\title{
Tumor promoting effects of CD95 signaling in chemoresistant cells
}

\author{
Elisabet Ametller1, Susana García-Recio', Domizziana Costamagna², Cristina Mayordomo ', Patricia Fernández- \\ Nogueira', Neus Carbó33, Eva María Pastor-Arroyo1, Pedro Gascón ${ }^{1}$ and Vanessa Almendro*1
}

\begin{abstract}
Background: CD95 is a death receptor controlling not only apoptotic pathways but also activating mechanisms promoting tumor growth. During the acquisition of chemoresistance to oxaliplatin there is a progressive loss of CD95 expression in colon cancer cells and a decreased ability of this receptor to induce cell death. The aim of this study was to characterize some key cellular responses controlled by CD95 signaling in oxaliplatin-resistant colon cancer cells.

Results: We show that CD95 triggering results in an increased metastatic ability in resistant cells. Moreover, oxaliplatin treatment itself stimulates cell migration and decreases cell adhesion through CD95 activation, since CD95 expression inhibition by siRNA blocks the promigratory effects of oxaliplatin. These promigratory effects are related to the epithelia-to-mesenchymal transition (EMT) phenomenon, as evidenced by the up-regulation of some transcription factors and mesenchymal markers both in vitro and in vivo.

Conclusions: We conclude that oxaliplatin treatment in cells that have acquired resistance to oxaliplatin-induced apoptosis results in tumor-promoting effects through the activation of CD95 signaling and by inducing EMT, all these events jointly contributing to a metastatic phenotype.
\end{abstract}

\section{Background}

CD95 (APO-1/Fas), a $48 \mathrm{kDa}$ membrane protein belonging to the TNF receptor superfamily, activates caspasedependent apoptosis in susceptible cells when is activated by its natural ligand (CD95L). There are two different cell types that use distinct CD95 apoptosis signaling pathways. After CD95 triggering, type I cells activate caspase8 at the death-inducing signaling complex (DISC) followed by activation of caspase-3. Apoptosis induction in these cells is not sensitive to mitochondria pathway inhibition. However, in type II cells DISC formation is strongly reduced and the activation of caspase- 8 and caspase-3 is produced downstream of mitochondrial events. Thus, inhibition of the mitochondrial role in these cells by overexpression of $\mathrm{Bcl}-2$ or $\mathrm{Bcl}-\mathrm{x}_{\mathrm{L}}$ blocks apoptosis $[1,2]$. This classification is related to cell phenotype, since type I cells correspond to mesenchymal tumors and type II cells display a more epithelial phenotype [3].

\footnotetext{
*Correspondence: almendro@clinic.ub.es

${ }^{1}$ Medical Oncology, Institut d'Investigacions Biomèdiques Agustí Pi y Sunyer (IDIBAPS), Institut Clínic de Malalties Hemato-Oncològiques (ICMHO), Hospital Clínic, Facultat de Medicina, Universitat de Barcelona, Spain

Full list of author information is available at the end of the article
}

Many cancer cells acquire survival advantage during tumor progression by decreasing its sensitivity to CD95induced apoptosis [4,5]. Some mechanisms affecting CD95 sensitivity include downregulation of CD95 protein expression [6], blocking of the active receptor site by the soluble form of CD95 ligand (sCD95L) [7], blocking of the interaction of CD95L with CD95 by the soluble decoy receptor 3 [8] and altered survival signaling pathways, among others (reviewed in [9]). In fact, this loss of CD95-responsiveness seems to be produced in parallel to tumor progression towards a more metastatic phenotype $[5,10]$. In colon cancer cell lines the functional elimination of CD95-responsive cells select for the outgrowth of different metastatic subpopulations, and cells isolated from the metastatic sites of xenografts tumors are resistant to CD95-induced apoptosis [10]. In agreement with this observation, there is almost a complete loss of detectable CD95 expression in metastatic lesions of colon cancer patients compared with the primary lesions [11].

However, this loss of sensitivity to CD95-induced apoptosis could reflect a change in its functionality since some of these CD95 apoptosis-resistant cells respond to CD95 activation with increased motility and invasiveness $[5,12]$, 
contributing to the development of the metastatic phenotype. In fact, it has been recently demonstrated that human and mouse colon cancer cells metastasize to the liver by using CD95 signaling [13]. Therefore, CD95 receptor can also exert roles beyond apoptosis and favor tumor-promoting effects in CD95 apoptosis-resistant tumor cells [12]. The selective pressure exerted by oxaliplatin treatment also selects for apoptosis-resistant cells with alterations in CD95-regulated signaling pathways and decreased CD95 expression. In these oxaliplatinresistant cells, activation of CD95 induces the phosphorylation of p42/44 MAPK and p38 MAPK, suggesting that changes in the receptor functionality are related to the acquisition of resistance to oxaliplatin [14]. In fact, induction of cell death by some cytotoxic drugs seems to be cell-type specific and to depend on the presence of an intact CD95 system [15]. For example, the apoptoticeffects of some cytotoxic agents such as doxorubicin and oxaliplatin are mediated by upregulation of CD95 and CD95L expression, and by activation of the apoptotic signaling in the neighboring cells [7]. Therefore, tumor progression or mechanisms of selective pressure that alter CD95 status would subsequently affect chemotherapy sensibility and cell behavior.

We previously reported that, in a way similar to what happens during tumor progression, acquisition of chemoresistance also selects for cells in which the functional activation of CD95 does not induced apoptosis but instead activates MAPK proteins and the NF- $\mathrm{kB}$ pathway [14]. Since oxaliplatin treatment is known to increase CD95 expression in both sensitive and resistant cell lines [14] the aim of the present study was to ascertain if oxaliplatin up-regulation of CD95 expression could contribute to the acquisition of a more aggressive behavior. Briefly, we show that drug treatment stimulates migration and decreases adhesion by means of CD95 activation in cells that have acquired resistance to oxaliplatin-induced death. We also report that oxaliplatin-resistant cells undergo EMT, as evidenced by the expression profile of several markers, and that oxaliplatin treatment also contributes to the acquisition of a more mesenchymal phenotype in the resistant cells and tumor xenografts. Finally, we have found that CD95 triggering in chemoresistant cells activates MAPK pathways and alters the expression levels of certain cell cycle proteins with previously described promigratory roles. We conclude that oxaliplatin treatment in cells that have acquired resistance to oxaliplatin-induced apoptosis could result in tumor-promoting effects by activation of CD95 promigratory signaling and by inducing EMT, which jointly contribute to the acquisition of a more metastatic phenotype.

\section{Results and Discussion}

\section{Oxaliplatin induces cell migration by activation of CD95} receptor in resistant cells

Some colon cancer cell lines resistant to CD95-induced apoptosis respond to CD95 activation with increased motility and invasiveness. Taking into account that oxaliplatin treatment increases CD95 expression in both sensitive and resistant cell lines, we aimed to determine if oxaliplatin stimulation of CD95 expression could exert a pro-migratory effect. To address directly this question we used a previously characterized model of acquired chemoresistance based on the cell lines HT29 and HCT116 $\mathrm{p} 53^{-/-}$and their corresponding oxaliplatin-resistant derived cell lines RHT29, and RHCT116 p53-/- [14]. To determine the effects of oxaliplatin and CD95 activation on cell migration we performed transwell migration assays as described in the Material and Methods section.

As expected, FBS stimulation induced a significant increase in basal cell migration both in HT29 and RHT29 cells (1,7 and 1,9-fold induction, respectively) and in the HCT116 p53-/- and RHCT116 p53/- (1,5-fold induction in both cell lines) (Figure 1A). Pretreatment of cells with oxaliplatin results in a higher migration capacity in RHT29 and RHCT116 p53/- cells (1,3-fold induction in both cases). Interestingly, a strong stimulation of cell migration was observed again in RHT29 and RHCT116 p53\% cell lines (3-fold and 1,5-fold induction, respectively) after agonistic $\mathrm{CH} 11$ antibody treatment (Figure 1A). This effect was not significantly enhanced when cells were pre-treated with oxaliplatin, probably due to the promigratory effects exerted by $\mathrm{CH} 11$ alone that masked the increase induced by oxaliplatin. On the other hand, neither HT29 nor HCT116 p53/- cells were affected by CH11-induced CD95 activation or drug pretreatment (Figure 1A), indicating that the activation of CD95 only induces cell migration in the oxaliplatin-resistant cell lines.

To better understand the contribution of CD95 in oxaliplatin-induced cell migration CD95 expression was abrogated by siRNA under the same experimental conditions (Figure 1A and Additional file 1: Fig S1). The inhibition of CD95 expression did not affect basal migration rate, but completely abrogated oxaliplatin-induced migration in the RHT29 and RHCT116 p53/- cell lines, confirming that the effects of oxaliplatin pretreatment in cell migration are mediated by the activation of CD95 receptor. Similar results were obtained when the cells were treated with the CD95-blocking antibody DX2 (Additional file 2: Fig S2). Finally, cell growth curves were performed under the same experimental conditions and no differences in cell proliferation rates were found (data 


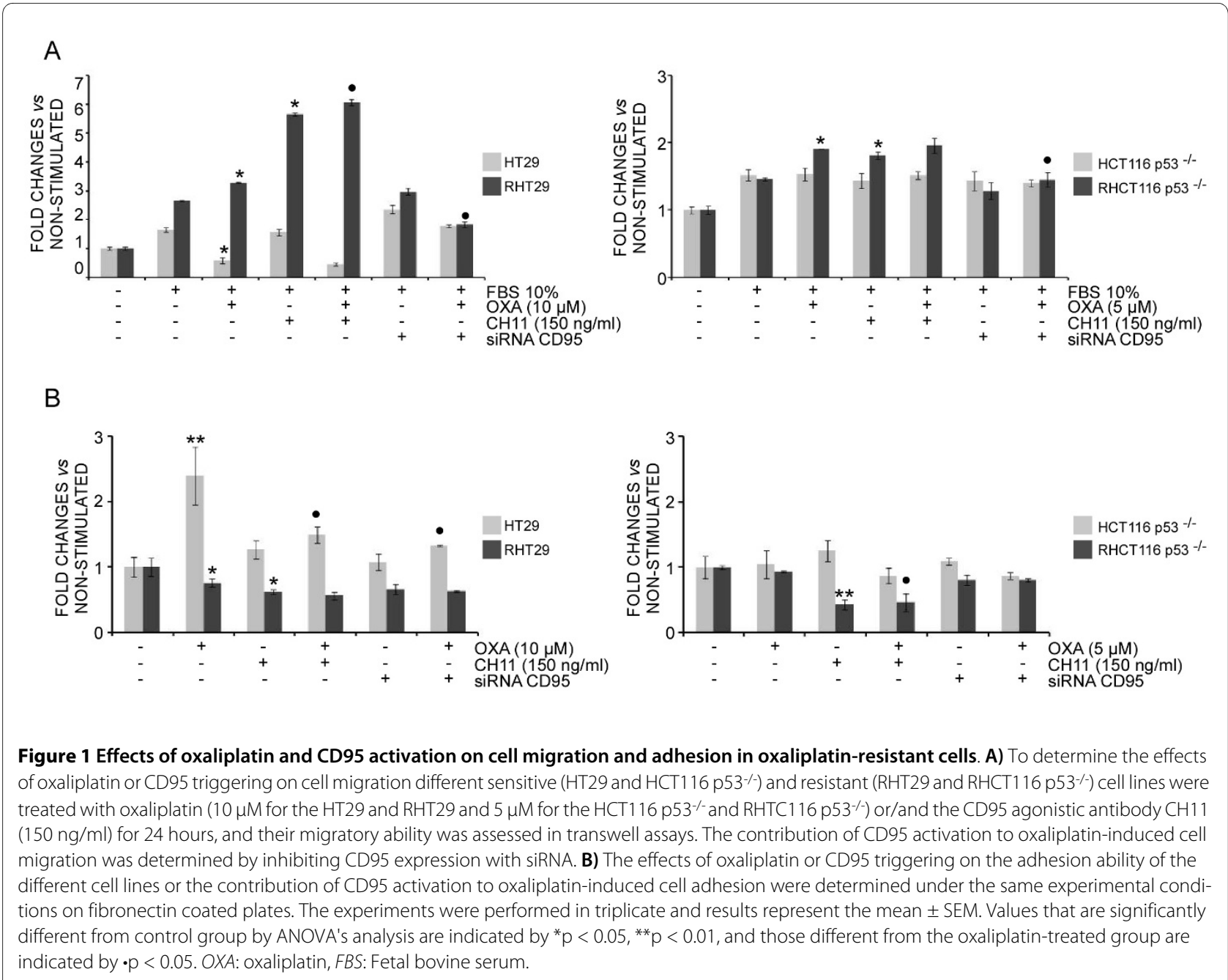

not shown), ruling out any possible contribution of differences in proliferation rates among cell lines to the migration assay results.

To determine if these effects on cell migration were accompanied by changes on cell adhesion we determined the capability of the various cell lines to adhere to fibronectin-coated surfaces. Oxaliplatin pretreatment significantly increased cell adhesion in HT29 cells but not in RHT29 cells (Figure 1B). Interestingly, however, CD95 stimulation by $\mathrm{CH} 11$ induced a significant decrease on basal adhesion ability in the RHT29 cell line and this was also evident even under oxaliplatin-pretreatment. CD95 inhibition by siRNA slightly affected RHT29 adhesion ability and significantly increased basal HT29 adhesion under oxaliplatin-pretreatment. Similar results were obtained when CD95 activation was blocked with DX2 antibody (Additional file 2: Fig S2). Finally, CD95 activation by $\mathrm{CH} 11$ also decreased cell adhesion in the RHCT116 $\mathrm{p} 53^{\%}$ cell line, while remaining unaltered by any treatment in HCT116 p53-/- cells (Figure 1B).
Taken together, these results indicate that oxaliplatin treatment stimulated cell migration in oxalipaltin-resistant cell lines by activating CD95 receptor, since forced activation of CD95 by an agonistic antibody strongly increased migration and decreased cell adhesion while CD95 silencing and inhibition efficiently abrogated oxaliplatin effects.

\section{Treatment with oxaliplatin induces the phosphorylation of p42/44 MAPK and $\mathrm{p} 38$ MAPK proteins}

Stimulation of motility and invasiveness by CD95 is mediated by the activation of different signaling pathways including caspase- 8 , NF- $\mathrm{kB}$ and phospho-p42/44 MAPK [12]. Moreover, oxaliplatin induces the activation of $\mathrm{p} 38$ MAPK [16] and NF-KB $[14,17]$ in several cell lines. The p38 MAPK has been described as a classical tumor suppressor [18,19], but p38 MAPK seems to play also other roles, such as increasing cell survival as a cellular defense mechanism to overcome the effects of cytotoxic drugs and also promoting cellular migration [20]. In addition, oxaliplatin increases NF-kB signaling in both HT29 and 
RHT29 cells but only the RHT29 cell line has dependence on the NF- $\mathrm{kB}$ pathway for survival [14]. For these reasons we next decided to determine if oxaliplatin treatment differentially contributes to the activation of p42/44 MAPK, p38 MAPK and the p65 subunit of NF-kB in the HT29 and RHT29 cell lines. We also investigated the signaling pathways activated by CD95 under oxaliplatin treatment.

HT29 and RHT29 cells were treated with oxaliplatin 10 $\mu \mathrm{M}$ for 48 hours and with $150 \mathrm{ng} / \mathrm{ml}$ of the CD95 agonistic antibody $\mathrm{CH} 11$ for 24 hours. As previously observed, oxaliplatin treatment activated p42/44 MAPK (Figure 2A) and p38 MAPK (Figure 2B). Activation of CD95 by $\mathrm{CH} 11$ increased the levels of p42/44 MAPK (Figure 2A) and p38 MAPK (Figure 2B) in the oxaliplatin-resistant cell line, but not in the sensible one. Moreover, oxaliplatin treatment slightly increased the effects of the agonistic antibody $\mathrm{CH} 11$ (Figure 2A and 2B). The inhibition of extracellular metalloproteinases, which decreased CD95 expression by receptor cleavage at the plasma membrane, also enhanced the effects of $\mathrm{CH} 11$ (data not shown), confirming the involvement of CD95 in the activation of MAPK pathways in the RHT29 cell line. Total amounts of active NF- $\mathrm{kB}$ were not significantly increased by CD95 activation in any of the cell lines analyzed (Figure 2C).

These results suggest that in cells that have acquired resistance to oxaliplatin CD95 triggering stimulates MAPK pathways known to contribute to the motility and invasiveness of these cells.

\section{Oxaliplatin treatment differentially affects cell cycle and apoptosis-related proteins}

$N F-\kappa B$ is constitutively expressed in a wide range of tumor cells, where it commonly promotes cell proliferation and resistance to apoptosis induced by genotoxic agents $[17,21]$. After exposure to oxaliplatin cells can suffer an arrest in G1 or G2/M followed by an S-phase delay [22-24]. Taking into account that cells respond to oxaliplatin by increasing NF- $\mathrm{KB}$ activity [14], which controls cyclin D1 expression [25], we also decided to analyze if oxaliplatin treatment was differentially affecting cell cycle profiles, proteins levels and apoptosis related proteins.

HT29 and RHT29 cell lines were treated with oxaliplatin for 24 and 48 hours and protein expression was determined by Western blot analysis. Basal levels of cyclin D1 were higher in the RHT29 cells compared to the HT29 cells (Figure 3A). Oxaliplatin treatment induced opposing effects in these cell lines. Whereas a significant increase in cyclin D1 expression was found in the RHT29 cell line, parental cells showed lower cyclin D1 protein expression after oxaliplatin. Interestingly, the upregulation of cyclin D1 expression in the RHT29 cells agrees with the increase in active NF- $\mathrm{kB}$ previously observed [14] and might be supporting their increased migratory ability described in the present paper (Figure 1A). Basal expres- sion of p27 was slightly lower in the RHT29 cell line, being not significantly affected by oxaliplatin treatment in any cell line.

Basal expression of CDK2 and CDK4 were significantly higher in the RHT29 cell line and oxaliplatin treatment increased CDK2 (transiently) and CDK4 expression (Figure 3A). CDK2 levels were found increased in HT29 cells after $48 \mathrm{~h}$ oxaliplatin treatment and a slight decrease was observed in CDK4 levels after $24 \mathrm{~h}$ of treatment in the HT29 cell line. Therefore, the RHT29 cell line showed higher levels of proteins related to proliferation and cell cycle progression and lower levels of the cell cycle inhibitor p27, all these changes accounting for its acquired resistance to oxaliplatin.

Next, we analyzed by flow cytometry the effects of 48 hours of oxaliplatin exposure $(10 \mu \mathrm{M})$ in the cell cycle distribution of both cell lines. The percentage of cells in the sub-G1 phase was significantly higher in the HT29 cell line compared to the RHT29 cell line, as expected (Figure $3 \mathrm{~B})$. In this case no arrest in the G1 phase was detected, probably because the dose and the time analyzed induced the majority of cells to undergo cell death. However, oxaliplatin induced an S-phase cell accumulation in the RHT29 cell line, suggesting that the drug could be promoting G1/S transition by upregulating cyclin D1, CDK4 and CDK2 but making it difficult to complete the Sphase, thus exerting a cytostatic rather than a cytotoxic effect on these oxaliplatin-resistant cells (Figure 3B).

We also determined the expression levels of active caspase-8, Bcl-2 and Bax proteins in HT29 and RHT29. We found that in the resistant cell line RHT29 oxaliplatin was less effective in inducing the activation of caspase-8 (Figure $4 \mathrm{~A}$ and $4 \mathrm{~B}$ ). Furthermore, we also found increased basal $\mathrm{Bcl} 2$ levels and an increased $\mathrm{Bcl} 2 / \mathrm{Bax}$ ratio in the oxaliplatin-treated RHT29 cells (Figure 4C-4E) further indicating that in this resistant cell line, the upregulation of survival pathways is contributing to the resistance to oxaliplatin-induced apoptosis.

\section{Acquisition of resistance to oxaliplatin induces EMT and confers a more motile phenotype}

The differential cell migratory responses of sensitive and resistant colon cancer cells to oxaliplatin led us to consider the possibility that resistant cells have undergone epithelial-to-mesenchymal transition (EMT), as described by others [26] and thus their motility capacity could result concomitantly affected. Gene expression of epithelial and mesenchymal markers and transcription factors related to the EMT reprogramming was studied under basal and oxaliplatin-treated conditions to determine if they were differentially expressed in HT29 and RHT29 cells. Figures 5 A1 and 5 A 2 show the differential basal expression of the EMT markers between HT29 and RHT29 cells. In spite of the lack of any significant 


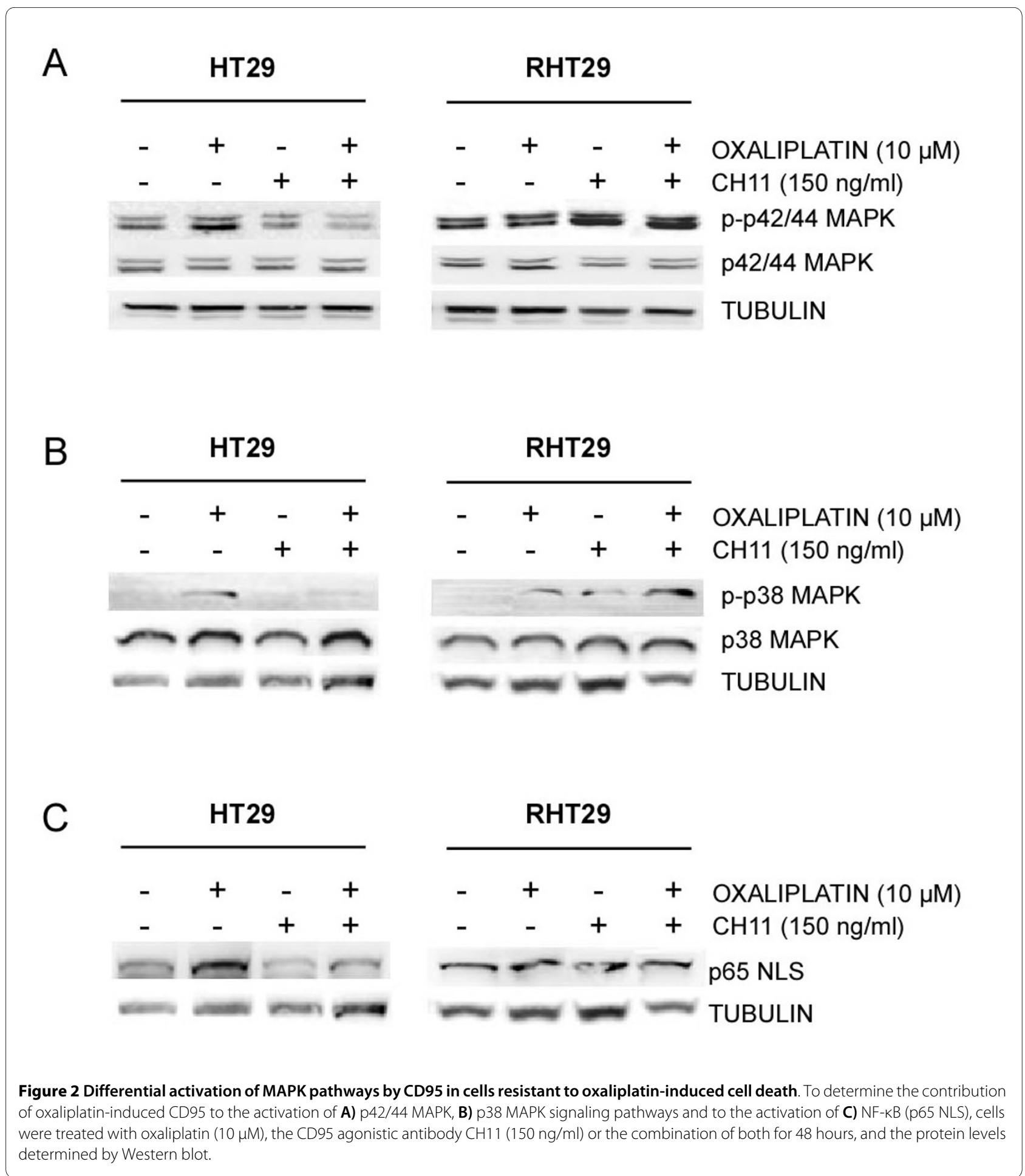

decrease in the epithelial markers E-cadherin, $\alpha$-catenin or $\gamma$-catenin, basal expression of the transcription factors Twist and Snail and the mesenchymal markers fibronectin and vimentin were significantly higher in the oxaliplatin-resistant cell line RHT29 (Figures 5 A1 and 5 A2), suggesting that parallel to the acquisition of chemoresistance there is a change in the expression profile of these markers towards a more mesenchymal phenotype [26]. Figures $5 \mathrm{~B} 1$ and $5 \mathrm{~B} 2$ show the differential induction of the same markers under oxaliplatin treatment in both cell lines, expressed as fold-induction versus basal levels. Although oxaliplatin failed to induce cell migration in the HT29 cell line, drug treatment up-regulated the expression of the transcription factors Twist, Snail and Slug, 


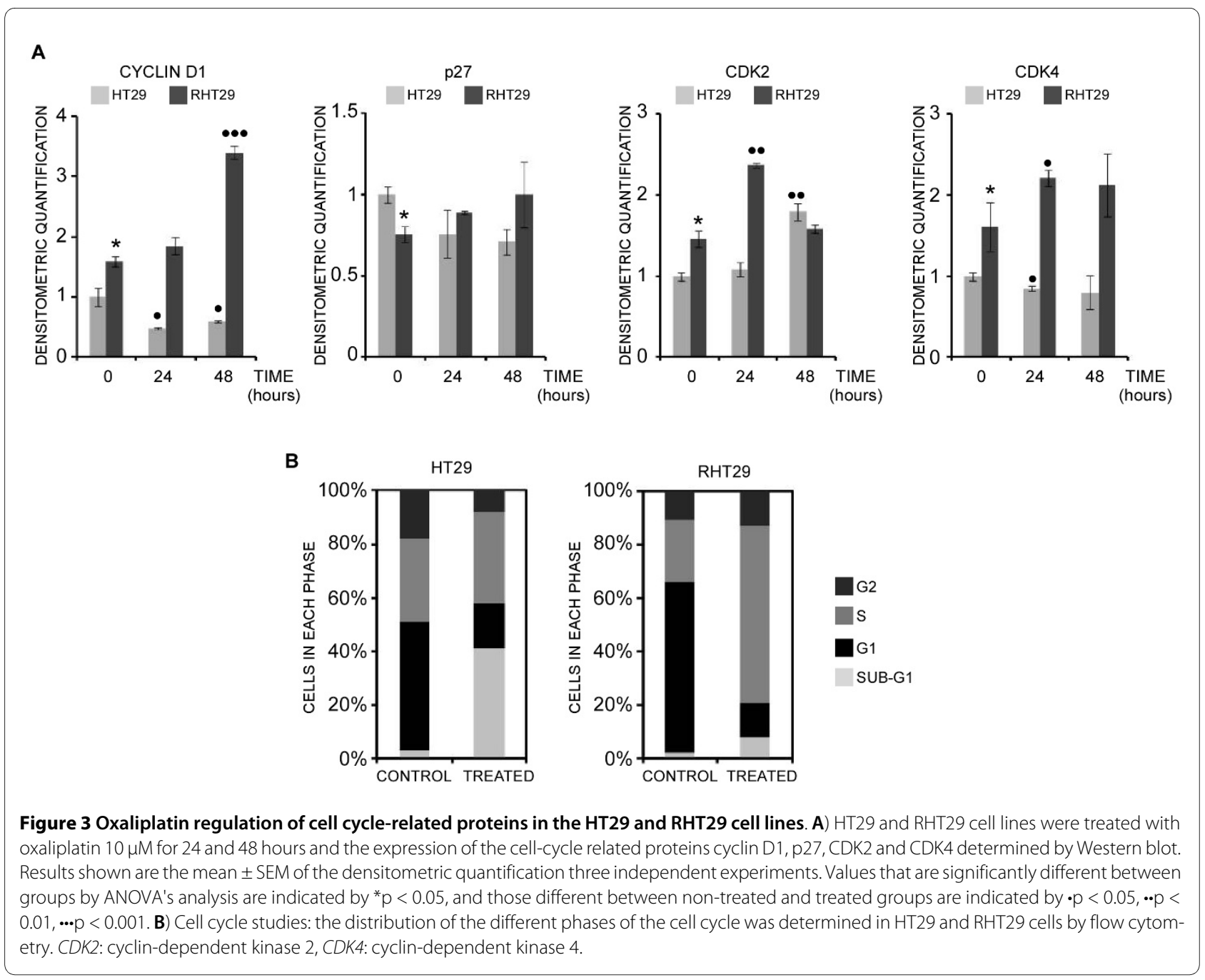

decreased $\alpha$-catenin expression and increased the mesenchymal markers fibronectin and vimentin (Figures 5 B1 and 5 B2). In the RHT29 cell line oxaliplatin seems to enhance the mesenchymal phenotype increasing the expression of some of the EMT markers such as Slug, fibronectin and vimentin and decreasing $\alpha$-catenin (Figures $5 \mathrm{~B} 1$ and $5 \mathrm{~B} 2$ ). In agreement with the stimulation of the EMT process, oxaliplatin treatment $(10 \mu \mathrm{M}$ for 24 hours) induced the development of a more fibroblast-like, spindle-cell morphology in both cell lines (Additional file 3: Fig S3).

We also determined the expression of E-cadherin by immunofluorescence and confocal microscopy analysis (Figure 5C). Interestingly, E-cadherin levels increased in the plasma membrane of the HT29 cells treated with oxaliplatin, in agreement with the slight increase on mRNA levels observed by qPCR. This could be related to the increased adhesion of the HT29 cells observed under oxaliplatin treatment (Figure 1B). Instead, basal E-cadherin expression progressively decreased in oxaliplatin- treated RHT29 cells (data not shown), being completely abrogated after 30 minutes (Figure 5C).

Finally, in an attempt to explore if this EMT process was also taking place in vivo, we analyzed the gene expression of the same markers in xenograft tumors, either treated with oxaliplatin or not, generated from the HT29 and the RHT29 cell lines [14]. Interestingly, similar changes in the basal gene expression pattern of the transcription factors, epithelial and mesenchymal markers related to the EMT process were observed in vivo between sensitive and resistant tumors when compared to the in vitro data (Figures $5 \mathrm{~A} 1$ and $5 \mathrm{~A} 2$ and Figure 6 A1 and 6 A2). Furthermore, oxaliplatin treated tumors closely mimic the changes on the expression patterns found in vitro (Figures $5 \mathrm{~B} 1$ and $5 \mathrm{~B} 2$ and Figure $6 \mathrm{~B} 1$ and 6 B2), further supporting the ability of oxaliplatin to induce EMT.

Therefore, oxaliplatin resistance induces the acquisition of a mesenchymal phenotype as observed for the RHT29 cell line and its xenograft tumor. Moreover, oxali- 
A

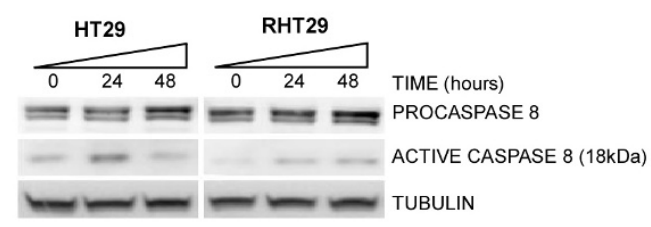

B

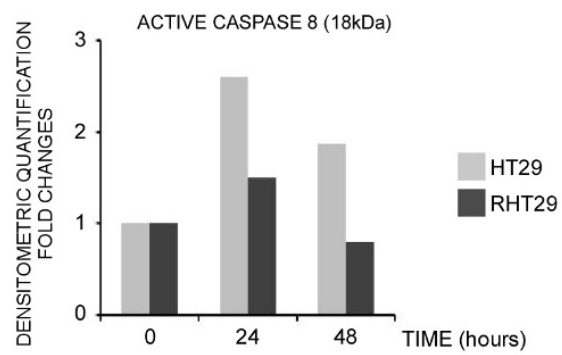

C

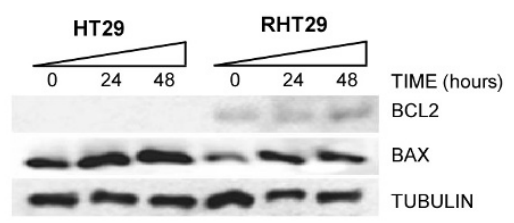

D

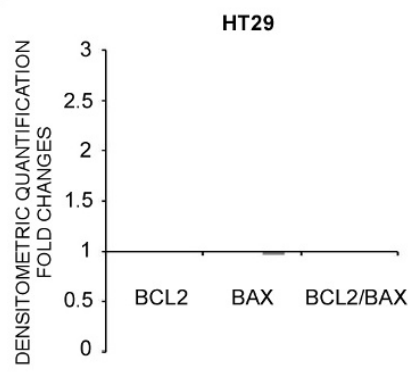

E

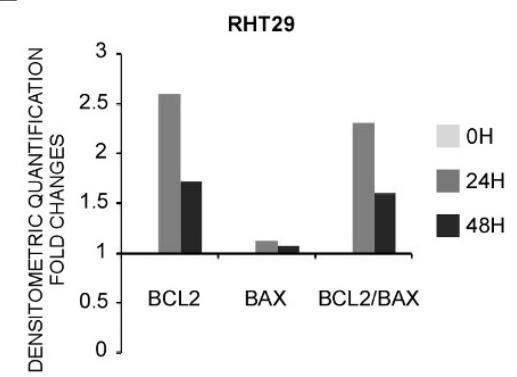

Figure 4 Oxaliplatin regulation of apoptosis-related proteins in the HT29 and RHT29 cell lines. HT29 and RHT29 cell lines were treated with oxaliplatin $10 \mu \mathrm{M}$ for 24 and 48 hours and the expression of the $\mathbf{A}$ ) active caspase 8 and $\mathbf{C}$ ) Bcl-2 and Bax were determined by Western blot. B) Densitometry of active caspase 8. D-E) Ratio between $\mathrm{BCl}-2$ and Bax for the HT29 and RHT29 cell lines based on the densitometry quantification.

platin treatment seems to induce gene reprogramming and EMT in both sensitive and resistant cells in vitro and in vivo that specifically confers a more motile phenotype to resistant cells.

\section{Conclusions}

In this study we have used previously characterized cell lines [14] as an in vitro model of acquired chemoresistance. We took advantage of the differential CD95 functionality displayed by these cell lines, as a result of the acquired chemoresistance process, to investigate the CD95-mediated cellular responses differentially regulated in sensitive and oxaliplatin-resistant cells. We have focused on the differential effects of oxaliplatin in the activation of MAPK pathways, migration and adhesion and the contribution of CD95 to all these processes, demonstrating that direct or oxaliplatin-induced CD95 activation in chemoresistant cells has tumor-promoting effects. Finally, we have demonstrated that oxaliplatintreated cells and tumors initiate but do not complete an EMT-like program and that chronic exposure to oxaliplatin renders them more prone to complete EMT, as they had already initiated EMT gene reprogramming during the acquisition of resistance to oxaliplatin. The diverse CD95-mediated oxaliplatin responses described can ultimately be responsible for the different cellular behavior observed between sensitive and resistant cells when exposed to oxaliplatin.

The general assumption that the CD95 receptor works as a death receptor has changed over the last years with the discovery that CD95 can mediate apoptosis-independent processes including proliferation, migration, angiogenesis and inflammation [12,27-29]. These changes in CD95 functionality seem to be related with membrane microdomain location, receptor internalization and endosomal trafficking [30]. However, whether the type of CD95 signaling is an intrinsic trait or a mechanism that can be acquired by changes in cell phenotype has never been addressed before. Moreover, specific tumor-promoting effects of CD95 stimulation in chemoresistant cells have not been reported. In cancer, the responsiveness to CD95 activation has been classified by Scaffidi $e t$ $a l$ [2] as dependent (type II cells) or independent (type I cells) from mitochondria. On the other hand, AlgecirasSchimnich et al [3] classified type I cell lines as those corresponding to a mesenchymal phenotype whereas the type II cell lines preferentially express epithelium-like markers.

The results from Algeciras-Schimnich et al [3] suggest that type I and II tumor cells represent different stages of carcinogenesis that resemble the EMT process. In fact, we have observed in our model of acquired chemoresis- 


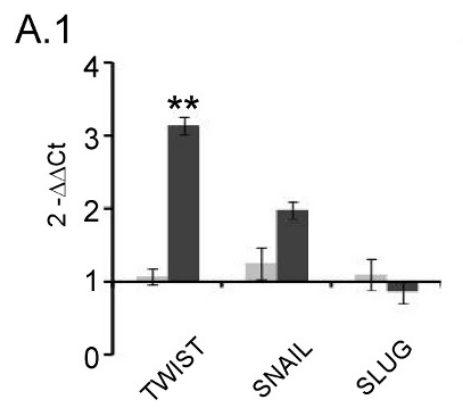

A.2

B.1

\section{B.2}
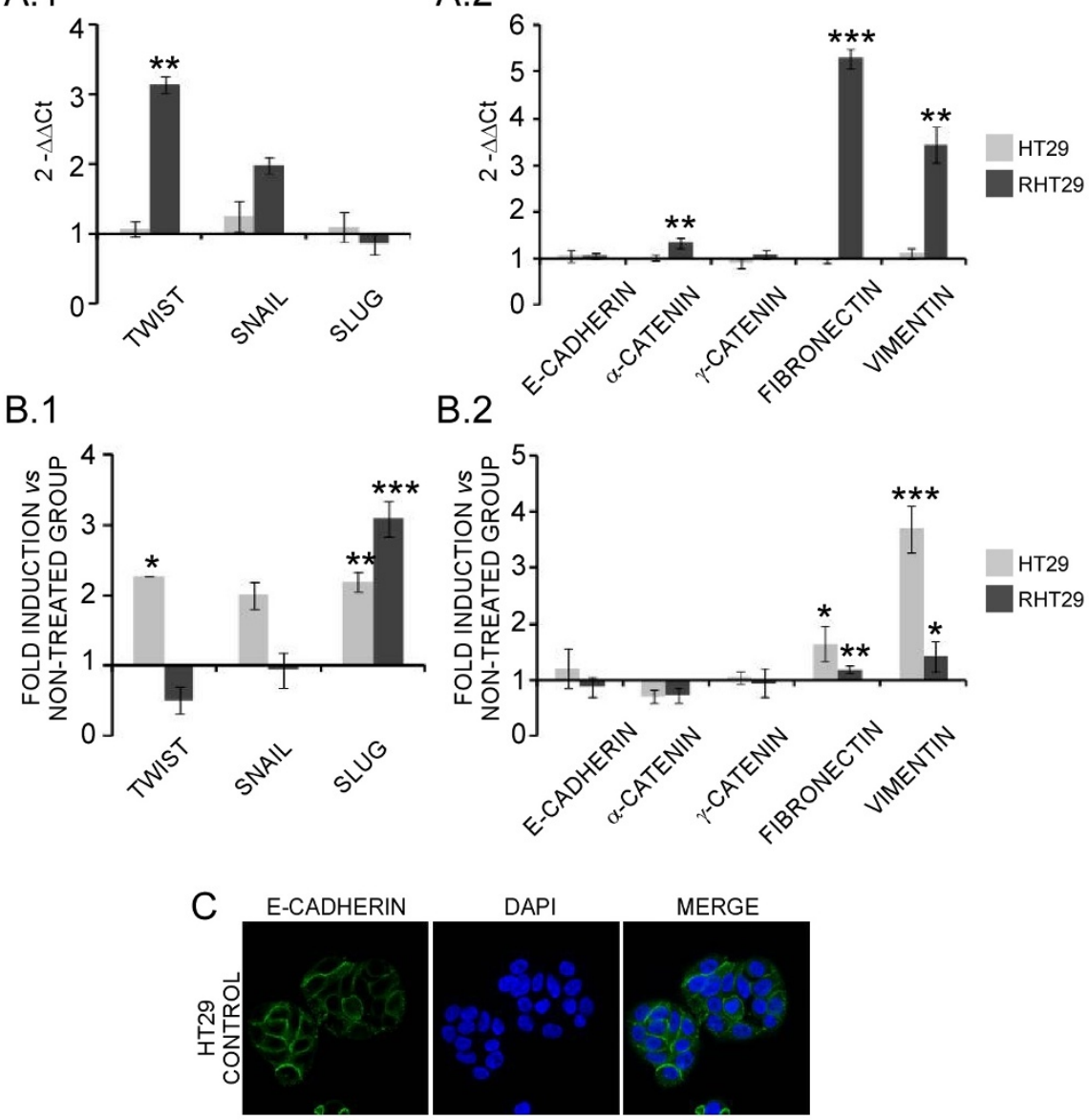

MERGE
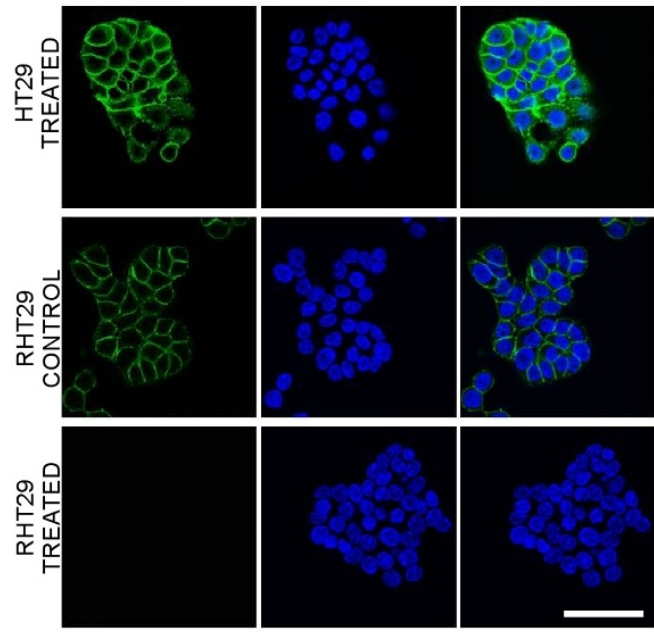

Figure 5 Analysis of EMT markers and E-cadherin expression on HT29 and RHT29 cells. (A1) Differential basal expression of some transcription factors related to the EMT process and (A2) markers of epithelial and mesenchymal phenotype were determined in the HT29 and RHT29 cell lines by qPCR. (B1) The effects of oxaliplatin in the gene expression of those transcription factors and (B2) EMT markers were also analyzed in cells treated with oxaliplatin $10 \mu \mathrm{M}$ for 48 hours and in this case the results are shown as fold induction versus non-treated cells. Results represent the mean \pm SEM of triplicates. Values that are significantly different between $(\mathbf{A})$ groups or between $(\mathbf{B})$ non-treated and treated groups by ANOVA's analysis are indicated by ${ }^{*} p<0.05,{ }^{* *} p<0.01,{ }^{* * *} p<0.001$. C) HT29 and RHT29 cells were treated with oxaliplatin $10 \mu \mathrm{M}$ for 48 hours and E-cadherin expression was detected by immunofluorescence and confocal microscope analysis. Scale bar is $50 \mu \mathrm{m}$. 


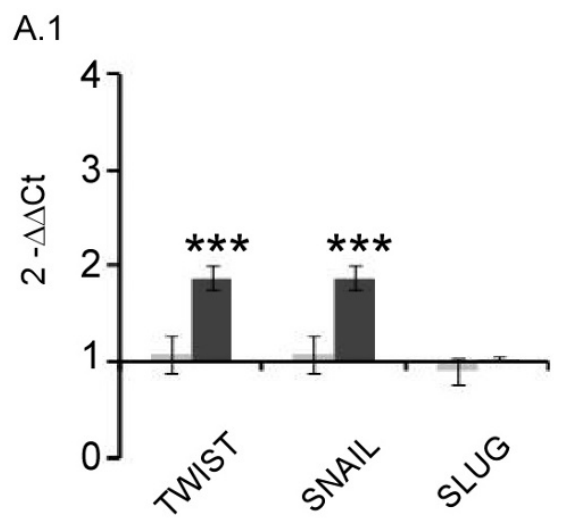

B.1

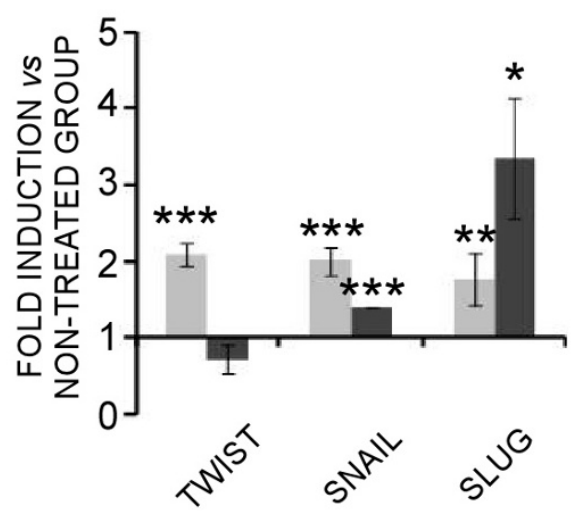

A. 2

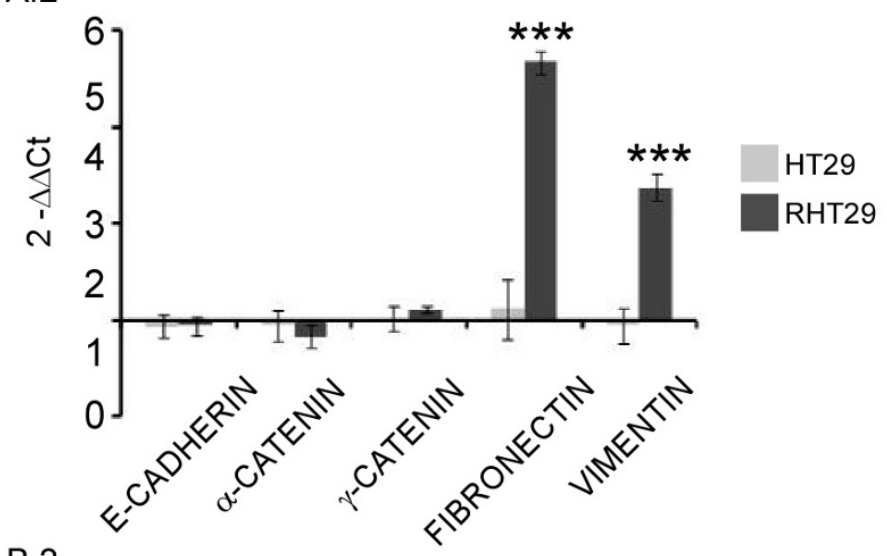

B.2

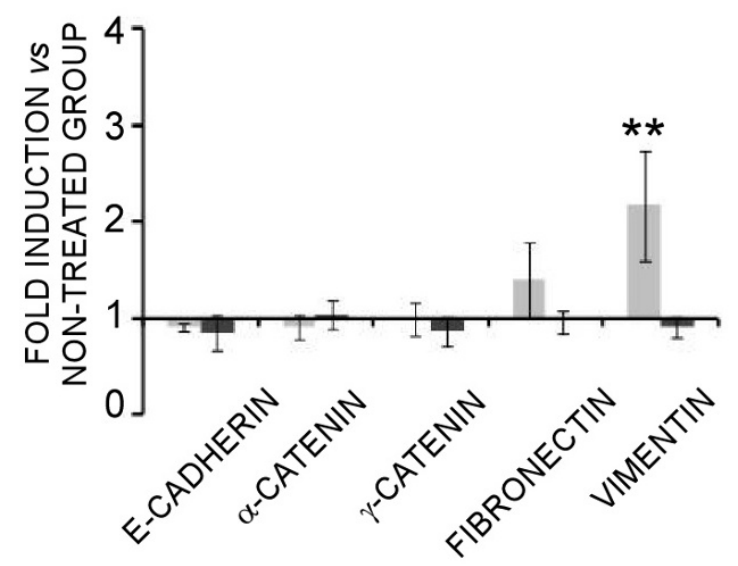

HT29

RHT29

Figure 6 Analysis of EMT markers on HT29 and RHT29 xenografts. (A1) Differential basal expression of some transcription factors related to the EMT process and (A2) markers of epithelial and mesenchymal phenotype were determined by qPCR in tumor xenografts derived from the HT29 and RHT29 cell lines. (B1) The effects of oxaliplatin in the gene expression of those transcription factors and (B2) EMT markers were also analyzed in xenografted tumors treated with oxaliplatin (i.p. $10 \mathrm{mg} / \mathrm{kg}$ ) once per week during 27 days and in this case the results are shown as fold induction versus non-treated tumors. Results represent the mean \pm SEM for 6 xenografts per group. Values that are significantly different between $(\mathbf{A})$ groups or between (B) non-treated and treated groups by ANOVA's analysis are indicated by ${ }^{*} p<0.05,{ }^{* *} p<0.01,{ }^{* * *} p<0.001$.

tance that the changes in CD95 signaling are accompanied by EMT, suggesting that CD95 functionality can switch as a consequence of changes in cell phenotype. Moreover, in these chemoresistant cells displaying a mesenchymal phenotype, CD95 signaling strongly induces cell migration and decreases cell adhesion in agreement with the proposed tumor-promoting effects of CD95 in some cell types.

The fact that during the acquisition of chemoresistance CD95 can switch its functionality could have relevant clinical implications. Chemotherapy acts as a selective pressure selecting resistant clones. If chemotherapy fails to eliminate the bulk of the tumor, the resulting resistant clones will sustain the regrowth of the tumor. As demonstrated in this work, these clones selected under chemotherapy show a mesenchymal phenotype and express a CD95 receptor with a non-apoptotic function. Moreover, the continuous exposure of these cells to oxaliplatin will allow the activation of CD95 and the stimulation of tumor-promoting pathways as well as cell migration. Therefore, oxaliplatin treatment of cells resistant to oxaliplatin-induced cell death could ultimately have tumor promoting effects by the upregulation and activation of prosurvival and promigratory CD95-controlled pathways. Then, more research efforts are needed to better understand how the acquisition of chemotherapy is produced, the time point at which tumor cells have acquired resistance to chemotherapy and consistently when the treatment could have detrimental effects.

\section{Materials and methods \\ Cell lines and reagents}

Human colon carcinoma cell line HT29 was purchased from American Type Culture Collection (Rockville, MD). 
The HCT116 $\mathrm{p}^{-1-}$ was generously given by Dr. Vogelstein. The oxaliplatin-resistant cell lines were developed by repeated exposure of the parental cells to increasing concentrations of oxaliplatin as we previously described [14]. Experiments were performed with the resistant population obtained from this process instead of using clones to better mimic the normal process by which a patient develops chemoresistance and to maintain some degree of tumor heterogeneity. All cell lines were cultured in McCoy's Medium supplemented with 10\% heat inactivated fetal bovine serum (FBS), antibiotics (Gibco, Invitrogen). The cultures were incubated at $37^{\circ} \mathrm{C}$ in a humidified $5 \% \mathrm{CO}_{2}$ atmosphere and the cells were serum starved overnight before experiments.

Antibodies used were mouse monoclonal anti-Fas (clone DX2) from Pharmingen; mouse monoclonal antiFas (clone $\mathrm{CH} 11$ ) from Upstate Biotechnology; mouse monoclonal anti-NF- $\mathrm{B}$ (NLS epitope) from Chemicon, rabbit polyclonal antibodies against phospho-p42/44 MAPK, p42/44 MAPK, phospho-p38 MAPK and p38 MAPK, were from Cell Signaling Technology, mouse monoclonal anti-caspase- 8 from Calbiochem, mouse monoclonal anti-Bcl2, rabbit monoclonal anti-Bax, mouse monoclonal anti-cyclin D1, rabbit polyclonal antibodies against CDK4, CDK2, and p27 were from Santa Cruz Biotechnology and mouse monoclonal anti-tubulin from Sigma. Oxaliplatin was from Sigma. All general reagents were purchased from Sigma, Bio-Rad and Amersham.

\section{Western blot}

The effects of oxaliplatin in regulatory cell cycle proteins and pro and anti-apoptotic proteins were detected by Western blot in cells treated with oxaliplatin $10 \mu \mathrm{M}$ during 24 and 48 hours. To study the contribution of CD95 to the induction of MAPK proteins and to the activation of NF- $\mathrm{kB}$ the colon cancer cell lines were treated with oxaliplatin $(10 \mu \mathrm{M})$ for 48 hours and with $\mathrm{CH}-11$ (150 ng/ $\mathrm{ml}$ ) for 24 hours. Protein was extracted and analyzed by Western blot as previously described [31] and tubulin expression was used as endogenous control. Image capture was performed with a Luminiscent Image Analyzer LAS-3000 (Fujifilm, Japan) and image analysis and densitometric quantification by using the Image Reader software (Fujifilm).

\section{Transfection with antisense CD95}

Silencing of CD95 was done by Stealth RNAi technology from Invitrogen as previously described for other genes [14], which provides three different chemical modified oligonucleotides against three different regions of the RNA target. The three probes used for the silencing of CD95 were: Probe 1, Cat\#10620318, FASHSS100597: UGAC AAUGUCCAAGACACAGCAGAA and Cat\#10620319
FASHSS100597: UUCUGCUGUGUCUUGGACAUUGUCA; Probe 2, Cat\#10620318 FASHSS100598: GGUU CUUACGUCUGUUGCUAGAUUA and Cat\#10620319 FASHSS100598: UAAUCUAGCAACAGACGUAAGA ACC; Probe 3, Cat\#10620318 FASHSS100599: GGGAU UGGAAUUGAGGAAGACUGUU and Cat\#10620319 FASHSS100599: AACAGUCUUCCUCAAUUCCAAUC CC. Before silencing experiments, the protocol was adjusted to ensure a minimum of $80 \%$ of RNA knockdown assessed by qPCR.

\section{Migration and adhesion}

Migration assays were performed in transwell inserts (with $8 \mu \mathrm{m}$ pore size and UV-opaque membrane from Becton Dickinson). The undersurface of transwells were coated with $5 \mu \mathrm{g}$ of human plasma fibronectin (SIGMA) or $1 \%$ BSA (negative control) and blocked with of $2 \%$ FBS. Cells previously treated with oxaliplatin oxaliplatin (10 $\mu \mathrm{M}$ for the HT29 and RHT29 and $5 \mu \mathrm{M}$ for the HCT116 p53\% and RHTC116 p53/-), CH-11 (150 ng/ml) or or DX2 $(400 \mathrm{ng} / \mathrm{ml})$ during 10 hours or silenced for CD95 by siRNA for 48 hours were labeled with Calcein-AM $5 \mu \mathrm{M}$ (Invitrogen) for $30 \mathrm{~min}$. Then, cells were resuspended in $100 \mu \mathrm{l}$ of serum-free medium and the corresponding treatment depending on the group (oxaliplatin, $\mathrm{CH}-11$ and DX2), and $130 \times 10^{3}$ cells per transwell were seeded and allowed to migrate for 14 hours at $37^{\circ} \mathrm{C}$ (time of every treatment at the end of the experiment was 24 hours). Medium containing a $10 \%$ of FBS was placed at the bottom of the wells and used as chemoattractant. After the 14 hours of migration cells on the bottom of the transwell were fluorimetrically detected by using the Biotek SpectraFluor plate reader, with $485 \mathrm{~nm}$ excitation and $530 \mathrm{~nm}$ emission filters. The experiment was performed in triplicate and fluorescence values were normalized versus the non-treated non-stimulated control cells.

For the adhesion assay 24-well plates were coated with fibronectin $(50 \mu \mathrm{g} / \mathrm{ml})$ or $1 \%$ BSA (negative control) at $37^{\circ} \mathrm{C}$ for 3 hours, and blocking of non-specific binding sites was performed using 1\% BSA during 30 minutes at $37^{\circ} \mathrm{C}$. Before assays, cells were treated with oxaliplatin oxaliplatin $(10 \mu \mathrm{M}$ for the HT29 and RHT29 and $5 \mu \mathrm{M}$ for the HCT116 p53/- and RHTC116 p53-/-), CH-11 (150 ng/ $\mathrm{ml})$ or DX2 $(400 \mathrm{ng} / \mathrm{ml})$ depending on the experimental group during 24 hours, or CD95 was silenced by siRNA. Cells were fluorescence-labeled with calcein-AM and $4 \times$ $10^{5}$ cells per well were left to adhered on the 24-well coated plates for 2 hours at $37^{\circ} \mathrm{C}$. Nonadherent cells were removed by washing the wells with $\mathrm{PBS}$ and attached cells were quantified by measuring the fluorescence emission using the Biotek SpectraFluor plate reader, with $485 \mathrm{~nm}$ excitation and $530 \mathrm{~nm}$ emission filters. Every experiment was performed in triplicate. 


\section{Immunofluorescence Assay}

For the detection of E-cadherin cells were seeded in coverslips until subconfluence and treated with oxaliplatin $10 \mu \mathrm{M}$ for 30 minutes. Then, cells were immunostained as previously described [14] using the mouse primary antibody against E-cadherin (Cell Signaling). Samples were viewed using an inverted epifluorescence microscope (Leica) and image assembly and processing were performed using the Image Processing Leica Confocal Software at the Microscopy Unit of the School of Medicine of the University of Barcelona.

\section{qPCR Analysis}

Total RNA from tumor cells was isolated with Ultraspec (Biotecx) according to the manufacturer's instructions. Quantitative PCR analysis was done on the ABI PRISM 7900 Sequence Detector System (Applied Biosystems) as previously described [14]. For determination of gene expression Assay-on-demand (Applied Biosystems) was used. Transcript levels were normalized to those of betaactin (Hs99999903_m1), which was used as endogenous control. Each experiment and every determination were done at least in triplicate, and the levels of gene expression were calculated using the $\Delta \Delta \mathrm{Ct}$ method.

\section{Cell cycle}

Cell cycle analysis was performed as described elsewhere in cells treated with oxaliplatin $10 \mu \mathrm{M}$ for $24 \mathrm{~h}$. DNA content was determined on a FACscan flow cytometer using FACSCalibur (BD Biosciences) and data processed by the software CellQuest. Every experiment was performed in triplicate.

\section{In vivo experiments}

In vivo experiments were performed with the HT29 and the RHT29 colon cancer cell lines as previously described [14]. All the experimental procedures with animals were performed in accordance with the regulations of our institution's Ethics Commission, following the guidelines established by the government of Catalonia, Spain.

\section{Statistical analysis}

Statistical analysis of the results was performed by ANOVA analysis. Statistical significance was considered for $\mathrm{p}$ values less than 0.05 .

\section{Additional material}

Additional file 1 Figure S1. Optimization of CD95 silencing by siRNA

Additional file 2 Figure S2. Effects of the CD95 blocking antibody DX2 on cell migration and adhesion.

Additional file $\mathbf{3}$ Figure S3. Effects of oxaliplatin in cell morphology.

The authors declare that they have no competing interests.

\section{Authors' contributions}

EA and SG performed the experiments, EA and DC did the EMT analysis by qPCR, CM and PF helped in the development of the chemoresistant cell lines, EP gave technical support, EA, NC, VA and PG discussed and interpreted the results, NC, EA and VA wrote the paper.

All authors have read and approved the final manuscript.

\section{Acknowledgements}

The authors acknowledge the technical assistance of Isabel Crespo. This work has been partially financed by grants from Fundación Cellex, by Fondo de Investigación Sanitaria, Instituto de Salud Carlos III (PI080022), by Redes Temáticas de Investigación en Cáncer (RTICC, RD07/0020/2014), by a grant from the Asociación Española Contra el Cáncer (AECC) (AECC_07_009) and by Generalitat de Catalunya, Catalonia, Spain (2009SGR624)

\section{Author Details}

'Medical Oncology, Institut d'Investigacions Biomèdiques Agustí Pi y Sunyer (IDIBAPS), Institut Clínic de Malalties Hemato-Oncològiques (ICMHO), Hospital Clínic, Facultat de Medicina, Universitat de Barcelona, Spain, 2Dipartimento di Medicina e Oncologia Sperimentale, Università di Torino, Italy and ¿Departament de Bioquímica i Biologia Molecular, Facultat de Biologia and Institut de Biomedicina (IBUB), Universitat de Barcelona, Spain

Received: 11 January 2010 Accepted: 23 June 2010

Published: 23 June 2010

\section{References}

1. Fulda S, Meyer E, Friesen C, Susin SA, Kroemer G, Debatin KM: Cell type specific involvement of death receptor and mitochondrial pathways in drug-induced apoptosis. Oncogene 2001, 20:1063-1075.

2. Scaffidi C, Fulda S, Srinivasan A, Friesen C, Li F, Tomaselli KJ, Debatin KM, Krammer PH, Peter ME: Two CD95 (APO-1/Fas) signaling pathways. EMBO J 1998, 17:1675-1687.

3. Algeciras-Schimnich A, Pietras EM, Barnhart BC, Legembre P, Vijayan S, Holbeck SL, Peter ME: Two CD95 tumor classes with different sensitivities to antitumor drugs. Proc Natl Acad Sci USA 2003, 100:11445-11450

4. Khong HT, Restifo NP: Natural selection of tumor variants in the generation of "tumor escape" phenotypes. Nat Immuno/ 2002 3:999-1005.

5. Peter $M E$, Legembre $P$, Barnhart $B C$ : Does CD95 have tumor promoting activities? Biochim Biophys Acta 2005, 1755:25-36.

6. Strand S, Vollmer P, van den Abeelen L, Gottfried D, Alla V, Heid H, Kuball J, Theobald M, Galle PR, Strand D: Cleavage of CD95 by matrix metalloproteinase-7 induces apoptosis resistance in tumour cells. Oncogene 2004, 23:3732-3736

7. Mitsiades N, Yu WH, Poulaki V, Tsokos M, Stamenkovic I: Matrix metalloproteinase-7-mediated cleavage of Fas ligand protects tumor cells from chemotherapeutic drug cytotoxicity. Cancer Res 2001 61:577-581

8. Pitti RM, Marsters SA, Lawrence DA, Roy M, Kischkel FC, Dowd P, Huang A, Donahue CJ, Sherwood SW, Baldwin DT, et al:: Genomic amplification of a decoy receptor for Fas ligand in lung and colon cancer. Nature 1998 396:699-703.

9. Igney $\mathrm{FH}, \mathrm{Krammer} \mathrm{PH}$ : Death and anti-death: tumour resistance to apoptosis. Nat Rev Cancer 2002, 2:277-288.

10. Liu K, McDuffie E, Abrams Sl: Exposure of human primary colon carcinoma cells to anti-Fas interactions influences the emergence of pre-existing Fas-resistant metastatic subpopulations. J Immuno/ 2003, 171:4164-4174

11. Moller P, Koretz K, Leithauser F, Bruderlein S, Henne C, Quentmeier A, Krammer PH: Expression of APO-1 (CD95), a member of the NGF/TNF receptor superfamily, in normal and neoplastic colon epithelium. Int J Cancer 1994, 57:371-377.

12. Barnhart BC, Legembre P, Pietras E, Bubici C, Franzoso G, Peter ME: CD95 ligand induces motility and invasiveness of apoptosis-resistant tumor cells. EMBO J 2004, 23:3175-3185.

13. Li H, Fan X, Stoicov C, Liu JH, Zubair S, Tsai E, Ste Marie R, Wang TC, Lyle S, Kurt-Jones E, Houghton J: Human and mouse colon cancer utilizes CD95 signaling for local growth and metastatic spread to liver. Gastroenterology 2009, 137:934-944. 944 e931-934. 
14. Almendro V, Ametller E, Garcia-Recio S, Collazo O, Casas I, Auge JM, Maurel J, Gascon P: The Role of MMP7 and Its Cross-Talk with the FAS/ FASL System during the Acquisition of Chemoresistance to Oxaliplatin. PLOS ONE 2009, 4:e4728.

15. Friesen C, Fulda S, Debatin KM: Cytotoxic drugs and the CD95 pathway. Leukemia 1999, 13:1854-1858.

16. Rakitina TV, Vasilevskaya IA, O'Dwyer PJ: Additive interaction of oxaliplatin and 17-allylamino-17-demethoxygeldanamycin in colon cancer cell lines results from inhibition of nuclear factor kappaB signaling. Cancer Res 2003, 63:8600-8605.

17. Wilson C, Purcell C, Seaton A, Oladipo O, Maxwell PJ, O'Sullivan JM, Wilson $\mathrm{RH}$, Johnston PG, Waugh DJ: Chemotherapy-induced CXC-chemokine/ CXC-chemokine receptor signaling in metastatic prostate cancer cells confers resistance to oxaliplatin through potentiation of nuclear factor-kappaB transcription and evasion of apoptosis. J Pharmacol Exp Ther 2008, 327:746-759.

18. Han J, Sun P: The pathways to tumor suppression via route p38. Trends Biochem Sci 2007, 32:364-371.

19. Hui L, Bakiri L, Stepniak E, Wagner EF: p38alpha: a suppressor of cell proliferation and tumorigenesis. Cell Cycle 2007, 6:2429-2433.

20. Thornton TM, Rincon M: Non-classical p38 map kinase functions: cell cycle checkpoints and survival. Int J Biol Sci 2009, 5:44-51.

21. Martinez-Balibrea E, Plasencia C, Gines A, Martinez-Cardus A, Musulen E, Aguilera R, Manzano JL, Neamati N, Abad A: A proteomic approach links decreased pyruvate kinase $\mathrm{M} 2$ expression to oxaliplatin resistance in patients with colorectal cancer and in human cell lines. Mol Cancer Ther 2009, 8:771-778

22. Noordhuis P, Laan AC, van de Born K, Losekoot N, Kathmann I, Peters GJ: Oxaliplatin activity in selected and unselected human ovarian and colorectal cancer cell lines. Biochem Pharmacol 2008, 76:53-61.

23. William-Faltaos $S$, Rouillard D, Lechat $P$, Bastian G: Cell cycle arrest by oxaliplatin on cancer cells. Fundam Clin Pharmacol 2007, 21:165-172.

24. Voland C, Bord A, Peleraux A, Penarier G, Carriere D, Galiegue S, Cvitkovic E, Jbilo O, Casellas P: Repression of cell cycle-related proteins by oxaliplatin but not cisplatin in human colon cancer cells. Mol Cancer Ther 2006, 5:2149-2157.

25. Miniati DN, Grunenfelder J, Hoyt EG, Murata S, Kown M, Koransky M, Robbins R: Oxidative stress of cardiac transplantation is associated with decreased BCL-2 expression and increased NFKB activity. J Heart Lung Transplant 2001, 20:158-159.

26. Yang AD, Fan F, Camp ER, van Buren G, Liu W, Somcio R, Gray MJ, Cheng H, Hoff PM, Ellis LM: Chronic oxaliplatin resistance induces epithelial-tomesenchymal transition in colorectal cancer cell lines. Clin Cancer Res 2006, 12:4147-4153.

27. Shinohara H, Yagita H, Ikawa Y, Oyaizu N: Fas drives cell cycle progression in glioma cells via extracellular signal-regulated kinase activation. Cancer Res 2000, 60:1766-1772.

28. Kaplan HJ, Leibole MA, Tezel T, Ferguson TA: Fas ligand (CD95 ligand) controls angiogenesis beneath the retina. Nat Med 1999, 5:292-297.

29. Biancone L, Martino AD, Orlandi V, Conaldi PG, Toniolo A, Camussi G: Development of inflammatory angiogenesis by local stimulation of Fas in vivo. J Exp Med 1997, 186:147-152.

30. Schutze S, Tchikov V, Schneider-Brachert W: Regulation of TNFR1 and CD95 signalling by receptor compartmentalization. Nat Rev Mol Cell Biol 2008, 9:655-662.

31. Lee KH, Feig C, Tchikov V, Schickel R, Hallas C, Schutze S, Peter ME, Chan $A C$ : The role of receptor internalization in CD95 signaling. EMBO J 2006, 25:1009-1023.

doi: 10.1186/1476-4598-9-161

Cite this article as: Ametller et al., Tumor promoting effects of CD95 signaling in chemoresistant cells Molecular Cancer 2010, 9:161

\section{Submit your next manuscript to BioMed Central} and take full advantage of:

- Convenient online submission

- Thorough peer review

- No space constraints or color figure charges

- Immediate publication on acceptance

- Inclusion in PubMed, CAS, Scopus and Google Scholar

- Research which is freely available for redistribution

Submit your manuscript at www.biomedcentral.com/submit
C Biomed Central 